\title{
Domain Pinning and Valence State Switching in $\mathrm{Pb}\left(\mathrm{Zr}_{0.2} \mathrm{Ti}_{0.8}\right) \mathrm{O}_{3} / \mathrm{La}_{0.8} \mathrm{Sr}_{0.2} \mathrm{MnO}_{3}$ Heterostructures
}

Dong Su, ${ }^{*}$ C. A. F. Vaz, ${ }^{* *}$ Y. Segal, ${ }^{* *}$ F. Walker,** C. H. Ahn, ${ }^{* *}$ M-G. Han, ${ }^{* * *}$ M. Sawicki, ${ }^{* * * *}$ and C. Broadbridge $* * * *$

* Center for Functional Nanomaterials, Brookhaven National Laboratory, Upton, New York, 11973

**Department of Applied Physics and Center for Research on Interface Structures and Phenomena, Yale University, New Haven, Connecticut 06520-8284

*** Condensed Matter Physics \& Materials Science Department, Brookhaven National Laboratory, Upton, New York, 11973

****Department of Physics, Southern Connecticut State University, $501 \mathrm{C}$ rescent Street, New Haven, CT, 06515

The creation of ferroelectric field effect transistors (FET) for nonvolatile memory elements can be achieved by the control of the spin state via electric fields in a multiferroic composition system [1]. For instance, in a bi-layer film composed of a ferroelectric perovskite and a colossal magnetoresistive $(\mathrm{CMR})$ manganite, it is possible to switch the spin state in CMR layer by controlling the pole states in the ferroeleetric perovskite. A recent report shows a s ignificant magneto-electric coupling in $\mathrm{PbZr}_{0.2} \mathrm{Ti}_{0.8} \mathrm{O}_{3} / \mathrm{La}_{0.8} \mathrm{Sr}_{0.2} \mathrm{MnO}_{3} / \mathrm{SrTiO}_{3}$ (PZT/LSMO/STO) heterostructures and the switching between the "depletion" and "accumulation" states is controlled by domain wall switching of the ferroelectric film [2]. In this presentation, we report TEM/STEM characterization of the $\mathrm{PZT} / \mathrm{LSMO} / \mathrm{STO}$ heterostructures.

We investigated the relationship between the domain feature and the switchability of the Mn valence, which is of importance for the performance of the devices. As shown in Figure 1, we found that the $90^{\circ}$ domains in PZT are non-switchable upon application of a $\pm 10 \mathrm{~V}$ electric field, applied using a single tilt Nanofactory holder. STEM-imaging shows that the $90^{\circ}$ domains have been pinned by a pair of misfit dislocations with Burgers vectors, $<100>$ and $<001>$ respectively. The pinning between the domain and the dislocation are analyzed using an effective dislocation model [3]. The dislocation dissociation of the $<110>$ dislocation is suggested to be coupled with the elastic domain formation. This finding indicates that the movement of $90^{\circ}$ domains in PZT islands is controlled by the removal of the dislocation pairs [4].

In the "depletion" and "accumulation" states, the valence state of $\mathrm{Mn}$ is supposed to be switched between a high spin state $\mathrm{Mn}^{3+}(\mathrm{S}=2)$ and a low spin state $\mathrm{Mn}^{4+}(\mathrm{s}=3 / 2)$ near the PZT/LSMO interface [2]. We use a STEM-EELS line-scan method to characterize the valence state change crossing the LSMO layer. As shown in Figure 2, the energy difference between $\mathrm{O}-\mathrm{K}_{\mathrm{a}}$ and $\mathrm{Mn}-\mathrm{L}_{3}$ edge shows an energy shift which is consistent with the prior results from $\mathrm{x}$-ray absorption spectroscopy[2].

\section{References}

[1] S. Mathews, R. Ramesh, T. Venkatesan, and J. Benedetto, Science 276, 238 (1997)

[2] C. A. F. Vaz, J . Hoffman, Y. Segal, J. W. Reiner, R. D. Grober, Z. Zhang, C. H. Ahn, and F. J. Walker, Phys. Rev. Lett. 104, 127202(2010)

[3] A. L. Roitburd, Phys. Stat. Sol. 37, 329 (1976) 
[4] V. Nagarajan, A. Roytburd, A. Stanishevsky, S. Prasertchoung, T. Zhao, L. Chen, J. Melngailis, O. Auciello, and R. Ramesh, Nature Mater. 2, 43(2003)

[5] T. Riedl, T. Gemming, W. Gruner, J. Acker, K. Wetzig, Micron, 38, 224(2007)

[6] Research carried out at the Center for Functional Nanomaterials, BNL, which is supported by the DOE, Office of Basic Energy Sciences, under Contract No. DE-AC02-98CH10886. We thank he financial support by the NSF through MRSEC DMR 0520495(CRISP), FENA and the NRI.
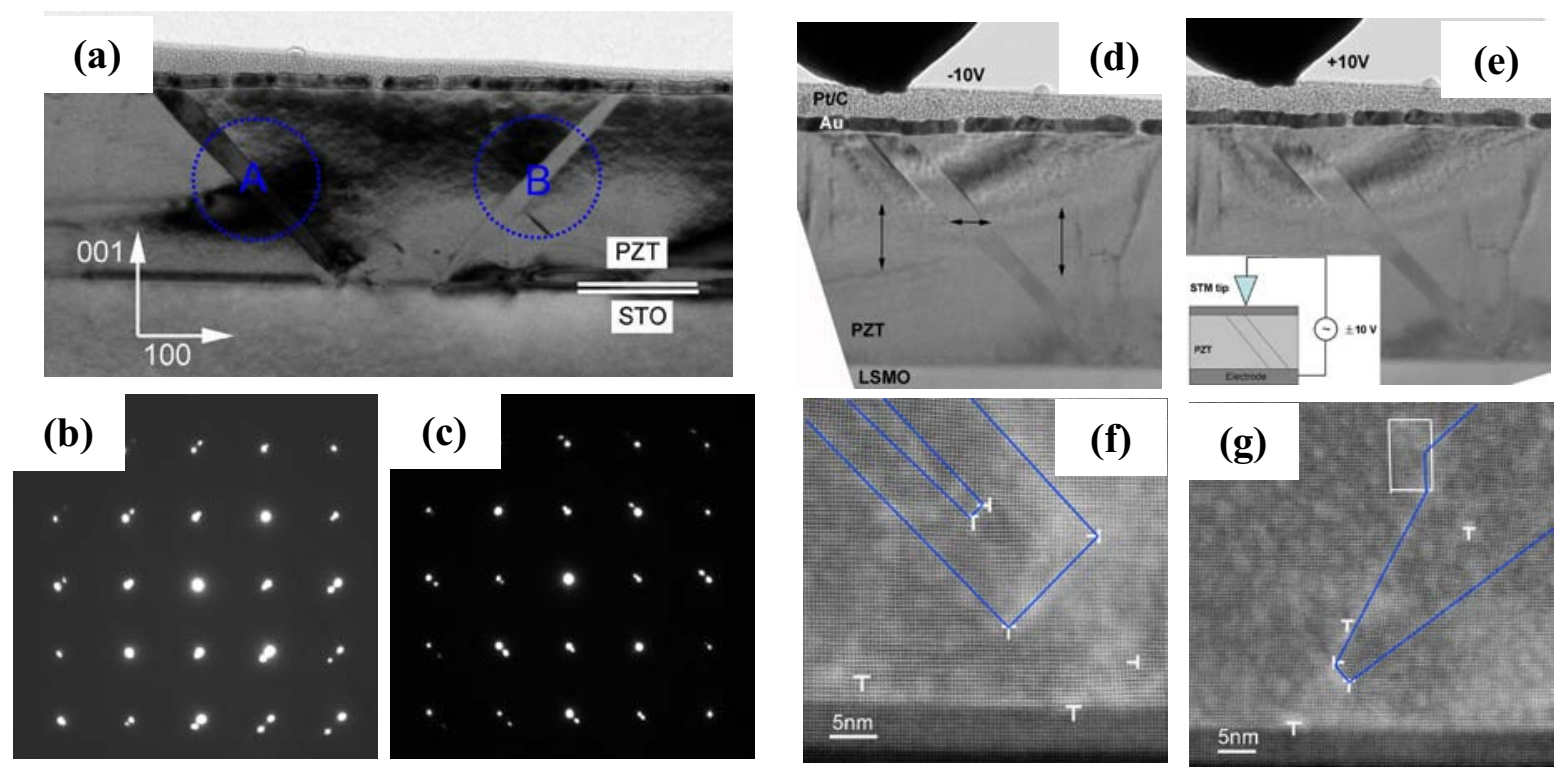

Figure1, (a) TEM bright-field image using $\mathrm{g}=00 \overline{2}$, showing two $90^{\circ}$ domains, marked by A and B respectively. (b) and (c) the corresponding SAED patterns from the circles around domain A and B in (a).(d) and (e) BF images showing A domain patterns under the switching voltages $10 \mathrm{~V}$ and $-10 \mathrm{~V}$. (f) and (g) ADF-STEM images on A and B domains respectively.
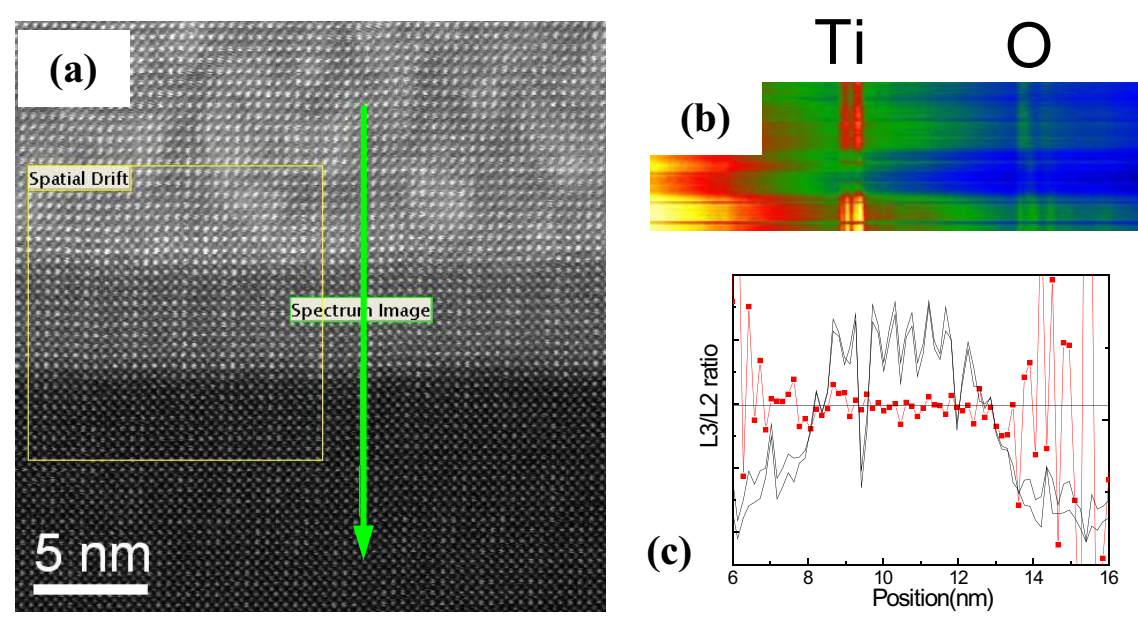

$\mathrm{Mn}$

Figure 2, (a) ADF-STEM image showing the interfaces between PZT, LSMO and STO. The scanning-line is indicated by the green line. (b) colorful spectrum-image of the line-scan showing the signals of Ti, $\mathrm{O}$ and $\mathrm{Mn}$. (c) Comparison of $\mathrm{Mn}$ signal and $\mathrm{Mn}_{3} / \mathrm{L}_{2}$ ratio (in red) as a function of position. (d) The energy difference between $\mathrm{O}-\mathrm{K}_{\mathrm{a}}$ and $\mathrm{Mn}-\mathrm{L}_{3}$ edges crossing the LSMO layer. 